\title{
Case Study: Pearls in Hypertension Pharmacotherapy
}

\author{
Jerome D. Cohen, MD, FACC, FACP, FAHA
}

\begin{abstract}
BACKGROUND: Research and therapy only has relevance when applied to an actual patient.

OBJECTIVE: To review a case study of a patient with hypertension and diabetes.

SUMMARY: The Seventh Report of the Joint National Committee on Prevention, Detection, Evaluation, and Treatment of High Blood Pressure (JNC 7) of 2004 recommends that a diagnostic workup include an assessment of risk factors and comorbidities using history, physical exam, and laboratory parameters. The presence of comorbidities influences drug selection. Patient evaluation should also include identification of possible causes of hypertension, such as renal arterial stenosis, and an assessment for the presence of target organ damage. Treatment is always influenced by the presence or absence of comorbidities. Lifestyle modifications are crucial to enhancing the success of pharmacologic therapy and should be ongoing. If lifestyle modifications do not work, the clinician must consider drugs. Study data and JNC 7 recommend beta-blockers for hypertension in patients with compelling indications, e.g., high risk for cardiovascular disease and diabetes.

CONCLUSION: JNC 7 emphasizes that evaluation for hypertension includes the assessment for the presence of compelling indications, e.g., diabetes, hyperlipidemia, and high coronary risk. These comorbidities may inform and direct pharmacologic choices.
\end{abstract}

KEYWORDS: JNC 7, Hypertension, Cholesterol, Diabetes, Blood pressure, Comorbidities, Lifestyle modification, Patient assessment

J Manag Care Pharm. 2007;13(5):S13-S16

\section{Author}

JEROME D. COHEN, MD, FACC, FACP, FAHA, is director, Preventive Cardiology Programs, and a professor of internal medicine, Division of Cardiology, Saint Louis University School of Medicine, Missouri.

AUTHOR CORRESPONDENCE: Jerome D. Cohen, MD, 8138 Westmoreland Ave., St. Louis, MO 63105. Tel: (314) 721-2820; Fax: (314)721-7976; E-mail: cohenjd@slu.edu

Copyright $@ 2007$, Academy of Managed Care Pharmacy. All rights reserved.
$\mathrm{R}$ esearch and theory only has relevance when one is talking about an actual patient. This case study describes a 76-yearold Hispanic woman, RM, who has type 2 diabetes in addition to hypertension. She also has peripheral artery disease and lower extremity edema. Her chief complaint is her blood pressure (BP) and some associated fatigue. Her systolic blood pressure (SBP) ranges from 140 to $200 \mathrm{~mm} \mathrm{Hg}$ and diastolic BP ranges from 70 to $104 \mathrm{~mm} \mathrm{Hg}$ when measured at home. Our patient is $5^{\prime} 2$ " and weighs 175 pounds. Her body mass index (BMI) is 32 and her waist circumference is 36". She is afebrile with a pulse of 70 . Her BP is elevated in the office at 160/84 mm Hg, even with repeated readings.

Her medications include the following:

- metoprolol XL 50 mg twice a day

- triamterene $37.5 \mathrm{mg} / \mathrm{HCTZ} 25 \mathrm{mg}$ once a day

- furosemide $40 \mathrm{mg}$ once a day

- olmesarten 20 mg every bedtime

- metformin 1 gram twice a day

- clonidine $0.2 \mathrm{mg} 4$ times a day and as needed

- aspirin $81 \mathrm{mg}$ once a day

- clopidogrel $75 \mathrm{mg}$ once a day

- ezetimibe $10 \mathrm{mg} / \mathrm{simvastatin} 40 \mathrm{mg}$ once a day

Despite these medications, her BP remains elevated.

During the past 4 weeks, her self-reported random blood glucose levels have ranged between 130 and $186 \mathrm{mg} / \mathrm{dL}$. She adheres to her diet but indicates that glucose control became a problem when the metoprolol XL was added to the regimen. She also complains of bouts of headaches and some facial redness, both of which signal an acute rise in BP, for which she takes clonidine $0.2 \mathrm{mg} 4$ times a day and as necessary for relief.

\section{Tailoring Care for the Hypertensive Patient}

The Seventh Report of the Joint National Committee on Prevention, Detection, Evaluation, and Treatment of High Blood Pressure (JNC 7) of 2004 recommends a diagnostic work-up beginning with an assessment of risk factors and comorbidities using history, physical exam, and laboratory parameters. ${ }^{1}$ Comorbidities present influence the drugs available for this patient. We attempt to reveal identifiable causes of hypertension, such as renal arterial stenosis. We also assess for the presence of target organ damage, particularly examining the eye carefully for hypertensive retinopathy; review the EKG for the presence of left ventricular hypertrophy (LVH), which suggests hypertensive heart disease, and review laboratory tests (urinalysis, blood glucose, hematocrit and lipid panel, serum potassium, creatinine, calcium; optional: urinary albumin/creatinine ratio, an assessment for renal disease) and a 12-lead electrocardiogram. ${ }^{1}$ Although JNC 7 indicates that the urinary albumin/creatinine ratio is considered optional, subsequent research has confirmed its importance in detecting early the presence of renal disease. 
In general, certain steps are essential when treating any patient with hypertension. Behavioral modification is often underemphasized. Weight loss with dietary restriction of simple carbohydrates and saturated fats is most important. In this diabetic patient, weight loss is critical because her glucose control is poor. Often, poor glucose control and poor BP control occur simultaneously. Weight loss is part of the standard treatment regimen and should be emphasized for every obese patient. Excess weight provides the underpinnings for metabolic syndrome: hypertension, dyslipidemia, and glucose intolerance or frank diabetes. It also indicates endothelial dysfunction. ${ }^{1}$

Increased physical activity—as appropriate for the patientis also critical. This 76-year-old woman could possibly increase her everyday physical activity by walking more and burning calories in other ways that are not arduous. This patient might be able to walk a few blocks or park the car a little farther away from a store on a nice day or walk down a flight of stairs rather than waiting for the elevator. Small energy expenditures in the form of brief exercise throughout the day over the long haul helps to control weight gain and can result in modest weight loss. Setting unattainable goals is an exercise in futility. ${ }^{1}$

Many patients are better able to stabilize their BP if they maintain a normal BMI $\left(18.5-24.9 \mathrm{~kg} / \mathrm{m}^{2}\right)$. Clinical trials have looked at the effect of weight loss on BP reduction. With each weight loss of 22 pounds (10 kilograms), SBP drops 5 to $20 \mathrm{~mm} \mathrm{Hg}$. Despite this significant improvement, the difficult, frustrating nature of weight loss sometimes discourages physicians and other health care providers from even recommending it. ${ }^{1}$

In terms of diet, the Dietary Approaches to Stop Hypertension (DASH) eating plan is a diet rich in fruits and vegetables enriched with low-fat dairy products as a source of calcium. This diet reduces total saturated fat. Adhering to the DASH program has resulted in SBP reductions in the range of 8 to $14 \mathrm{~mm} \mathrm{Hg}$. Recommending dietary sodium reduction is important for all hypertensive patients, even though most people will respond, "I don't eat salt, I don't add it at the table, and I don't cook with it." Heightening their awareness that $70 \%$ of the sodium we consume is derived from processed foods and teaching them how to read labels will help, as will directing them to low-sodium products available as alternatives for most foods. Sodium reduction can lower BP an additional 2 to $8 \mathrm{~mm} \mathrm{Hg}$ above that achieved with weight loss. ${ }^{1}$

Alcohol restriction or reduction is important for those who have a more than moderate intake ( 2 drinks per day for men, 1 per day for woman). When taken to excess, alcohol can contribute to high $\mathrm{BP}$ and is also associated with poor adherence to medical regimens. ${ }^{1}$

Basically, the goal is a stable, consistent foundation of a prudent lifestyle consuming fewer calories, increasing physical activities when possible, and making good lifestyle choices. The particular patient in this case study is high risk, having hypertension, diabetes, and dyslipidemia. Appropriate lifestyle modification can address all 3 problems.

\section{Selecting Appropriate Pharmacotherapy}

Treatment is always influenced by the presence or absence of comorbidities. If lifestyle modifications do not work, the clinician must consider drugs. The figure demonstrates the steps the clinician would consider for active drug treatment, according to JNC $7 .{ }^{1}$

JNC 7 stages hypertension, and an SBP of 140 to $159 \mathrm{~mm} \mathrm{Hg}$ is considered stage 1 hypertension; JNC 7 recommends monotherapy initially. A thiazide, angiotensin-converting enzyme inhibitor (ACEI), beta-blocker, calcium channel blocker (CCB), or a combination, if necessary, is appropriate as a first step. In an uncomplicated patient, the goal is usually less than $140 \mathrm{~mm} \mathrm{Hg}$ systolic and less than $90 \mathrm{~mm} \mathrm{Hg}$ diastolic. Generally, the lower the pressure, the better; there is nothing magical about 139/89 mm $\mathrm{Hg}$. Using lifestyle intervention with drug therapy can help move the BP reading more toward an optimal reading. ${ }^{1}$

Patients with stage 2 hypertension (SBP $>160 \mathrm{~mm} \mathrm{Hg}$ ) may require treatment with 2 drugs, even initially. Usually, a thiazide is included in 2 drug regimens because it works well in combination with other antihypertensives, including beta-blockers, angiotensin II receptor blockers (ARBs), and ACEIs or CCBs. Clinical judgment is important, as is awareness of comorbid conditions.

Patients who have compelling indications for specific drug classes are handled differently. Compelling indications are those indications in which benefit from a specific agent has already been shown. An example is beta-blockers' association with a reduction in myocardial infarction (MI). Clinicians choose a beta-blocker for patients with histories of MI to capitalize on its ability to reduce the likelihood of recurrent MI and to lower BP.

If the patient's BP does not reach the goal, we will perhaps increase the dose (if it is not already at the maximum recommended dose) and/or add another agent.

A meta-analysis of trials $(\mathrm{N}=18,883)$ investigating the use of beta-blockers in hypertension was conducted in 1997. Beta-blockers JNC 7: Tailor Treatment to Comorbidities

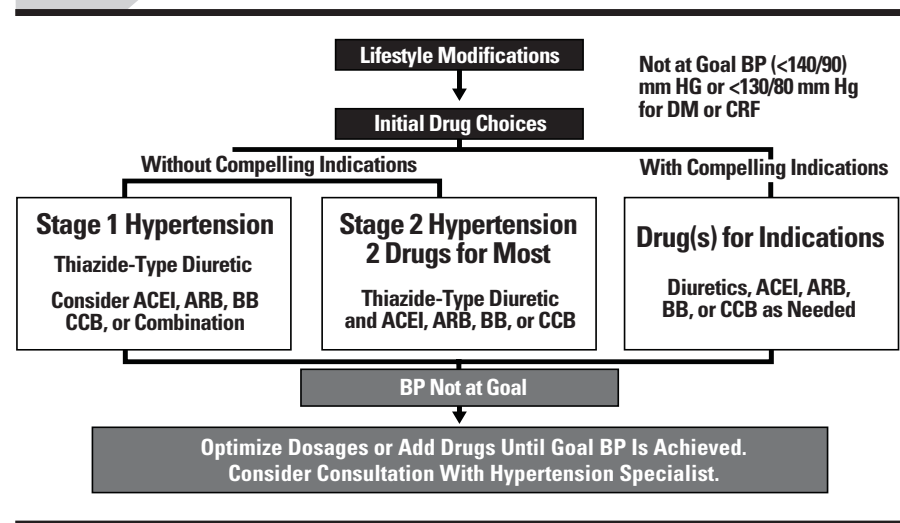

$A C E I=$ angiotensin-converting enzyme inhibitor; $A R B=$ angiotensin II receptor blocker; $B B=$ beta blocker $; C C B=$ calcium channel blocker. 
were associated with a reduction in cardiovascular disease (CVD). Psaty et al. identified a significant reduction in heart-failure (42\%) and stroke (29\%) and a less robust reduction in coronary events (7\%), which was not quite statistically significant. These findings make sense because stroke and heart failure are closely correlated to hypertension. The correlation coefficient of BP to heart failure and stroke is much greater than the correlation coefficient with coronary disease. ${ }^{2}$

Beta-blockers are used less in hypertensive patients with diabetes, kidney disease, or stroke. Total beta-blocker use in hypertension with heart failure is 56\%, which reflects a need for increased understanding by physicians of the importance of these agents. Part of the problem rests with the historical contraindication of betablocker use in patients with heart failure. This contraindication has been put to rest, and we know that reducing the sympathetic responsiveness in heart failure can reduce adverse cardiac events. Three quarters of prescribers use beta-blockers as an intervention for compelling indications in the post-MI patient. In patients with high risk for CVD who do not have heart failure or have had an MI, the use of beta-blockers lags at $37 \% .^{3}$

Clinical trials and guidelines for compelling indications for individual drug classes indicate when diuretics, beta-blockers, ACEIs, ARBs, CCBs, and aldosterone antagonists should be employed. For beta-blockers, the best candidates are heart-failure patients (in whom beta-blockers are, in fact, a standard of care); post-MI patients to reduce the likelihood of recurrent MI; highrisk coronary patients to improve endothelial function; and in highest-risk patients (diabetics). ${ }^{1}$

Regulatory agencies have approved beta-blockers for a remarkable number of indications, but beta-blockers can be

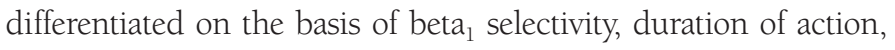
intrinsic sympathomimetic activity, lipophilicity, and whether the beta-adrenergic blocking action is accompanied by an alphaadrenergic blocking action. Nebivolol's ability to activate nitric oxide (NO) synthetase in blood vessels is a unique therapeutic option. It may be useful in a range of off-label indications. ${ }^{4}$ Its utility in high-risk patients appears to be quite broad. Heart failure, prolonged QT interval syndrome (a common cause of sudden death), and myopathies are just a few possible conditions where it may be useful; however, it must be emphasized that these are not approved indications. ${ }^{4}$

Primary care physicians' perception of antihypertensives' effectiveness can be seen in their prescription choices. For physicians who tend to prescribe ACEIs or CCBs first, these therapies are perceived to be effective in $62 \%$ and $58 \%$ of patients, respectively. For beta-blocker and diuretic therapies, the physicians' perceived effectiveness was $55 \%$ and $39 \%$, or about the same as for those physicians who did not prescribe these agents first. This is not a statistically significant difference. Evaluating the $P$ values for betablockers $(P=0.46)$ and for diuretics $(P=0.12)$ shows that because the ACEI and CCB difference is statistically significant for perceptions by physicians playing a role in their initial choice of

\section{TABLE \\ Perceived Effectiveness of Antihyper- tensives by PCPs by Prescription Choice $^{5}$

\begin{tabular}{c|c|c|c}
\hline & $\begin{array}{c}\text { Physicians } \\
\text { Prescribing ACEI } \\
\text { or CCB } \\
(\mathrm{N}=190)^{*}\end{array}$ & $\begin{array}{c}\text { Physicians } \\
\text { Prescribing BB } \\
\text { or Diuretic } \\
(\mathrm{N}=176)^{*}\end{array}$ & $\begin{array}{c}\text { PValue } \\
\text { Comparison } \\
\text { Between } \\
\text { Physician Group }\end{array}$ \\
\hline
\end{tabular}

Effectiveness

[Mean (SD) \% of time drug expected to be effective]

\begin{tabular}{l|c|c|c}
\hline ACEI & $62(23)$ & $49(27)$ & $<0.001$ \\
\hline BB & $53(26)$ & $55(24)$ & 0.46 \\
\hline CCB & $58(24)$ & $51(27)$ & 0.006 \\
\hline Diuretic & $35(23)$ & $39(26)$ & 0.12 \\
\hline
\end{tabular}

${ }^{*} N=$ total of effectiveness and tolerability data.

$A C E I=$ angiotensin-converting enzyme inhibitor; $B B=$ beta blocker; $C C B=$ calcium channel blocker, $P C P=$ primary care physician.

therapy, the effectiveness of the drugs eventually influences their decision regardless of what they believe initially. If a physician really believes something is going to work, he or she will use it. Through education, physicians will understand that older drugs, like beta-blockers and diuretics, still have an important role in treating hypertension. Newer agents, and particularly the beta-blocking agents, actually can improve endothelial function and should be considered; that opportunity wasn't available in the past (Table). ${ }^{5}$

JNC 7 emphasizes that evaluation for hypertension includes assessing for the presence of compelling indications; e.g., diabetes, hyperlipidemia, and high coronary risk. These comorbidities may determine specific pharmacologic choices. Lifestyle modifications are crucial to enhancing the success of pharmacologic therapy and should be ongoing. Study data and JNC 7 recommend betablockers for hypertension in patients with compelling indications; e.g., at high risk for CVD and diabetes.

Edgar R. Gonzalez, PharmD, served as moderator for this program (see Faculty).

Dr. Gonzalez: Many years ago, the JNC promoted the statement, "Let's go with step care." Step care promulgated diuretics as first-line treatment, beta-blockers as second line, and newer agents as third line. Then, a later version of JNC proposed making step care less rigid. Are we seeing a rebirth of beta-blockers? And why has step care been deemphasized?

Dr. Cohen: Step care was based on Veterans Administration [VA] studies that demonstrated the benefit of lowering blood pressure. The VA started with a diuretic and added reserpine if necessary. Beta-blockers were not available. Hydralazine was used as a vasodialator, and guanethidine was the fourth-step drug. All these drugs have, for the most part, outlived their utility. Applying the VA algorithm for uncontrolled hypertension was the standard for 10 to 12 years. Many physicians labeled it "cookbook medicine" and did not use step care. 
Medicine's advances brought new pharmacologic agents: betablockers, CCBs, ACEIs, and most recently, ARBs. This expanded armamentarium and the clinical trials provided the basis for making specific recommendations with respect to compelling indications. JNC guidelines, using the evidence available, evolved. For some patients, nonpharmacologic treatment is appropriate and effective. Allowing an element of clinical judgment persuaded many physicians to conduct careful history, physical, and laboratory assessments to determine which drug to use.

Current medical practice incorporating evidence-based treatment guidelines indicates that patients are being treated more appropriately and control of hypertension is better overall. We now understand that lowering SBP below $140 \mathrm{~mm} \mathrm{Hg}$ is better, but we are not necessarily satisfied with a target of $139 \mathrm{~mm} \mathrm{Hg}$. In the diabetic patient, for example, and now the renal patient, we understand that we should have an often difficult-to-achieve goal of less than $130 \mathrm{~mm} \mathrm{Hg}$. Some of the older drugs, namely, diuretics and beta-blockers and particularly the third-generation beta-blockers, have a lot to offer in terms of maximizing blood pressure reductions and providing benefits beyond just the blood pressure lowering.

Dr. Gonzalez: You were involved with JNC 7 and you have a great understanding of the Multiple Risk Factor Intervention Trial. Is it fair to say that MRFIT opened our eyes to the fact that blood pressure is just not a value or an independent number?

Dr. Cohen: That's true. Medical professionals now see the continuous relationship between blood pressure and risk for heart attack, stroke, and heart failure. An SBP of 100 plus the patient's age used to be considered acceptable. The MRFIT and Systolic Hypertension in the Elderly Program [SHEP] studies using diuretics and beta-blockers demonstrated that prudent drug use could contribute to a significant reduction in morbidity and mortality. ${ }^{6-8}$ Subsequently, heart failure admissions to hospitals, the number 1 diagnosis-related group in this country for Medicare patients, fell 54\%, strokes were reduced by 33\%, and heart attacks fell approximately $20 \% .^{9}$

Higher BP over time is not something we should write off to old age. It is a treatment target because the risk of stroke, heart attack, and heart failure is real. Patients fear strokes. This fear often persuades them to take medications and may improve adherence.

Dr. Gonzalez: You've served on JNC 7 and have brought us up-todate on the knowledge of NO synthetase. Why have the British downplayed beta-blockers in their National Institute for Health and Clinical Excellence (NICE) guidelines?

Dr. Cohen: The British scientists, using a study they conducted called Anglo-Scandinavian Cardiac Outcomes Trial (ASCOT), found that the blood-pressure-lowering arm that used a CCB and an ACEI versus a beta-blocker (atenolol) and a diuretic did better. ${ }^{10}$ But the data show that, in compelling indications populations, beta-blockers are the drugs of choice for the high-risk patient. Vasodilating ability can not only lower blood pressure but can also improve endothelial function and thereby reduce morbidity and mortality. Beta-blockers are an important class of drugs to use or consider using in the hypertensive patient with comorbidities and in those patients who are at high risk.

Dr. Gonzalez: In essence, the data from the Glycemic Effects in Diabetes Mellitus Carvedilol-Metoprolol Comparison in Hypertensives [GEMINI] trial and some newer studies show that not all beta-blockers are created equal; some third-generation beta-blockers may not mimic what atenolol did in the ASCOT trial. In the future, we may increase our dependency on beta-blockers in hypertension in the patient with multiple risk factors.

Dr. Cohen: Yes, the ASCOT study used atenolol, but in the post-MI studies, atenolol was never used. Those studies used propranolol, metoprolol, and timolol. Timolol is now used predominantly in ophthalmic preparations. Atenolol is short acting and has not been studied for MI, and that is why we do not use it. We often discuss actions and effects of beta-blockers and call them class effects. The second- and third-generation drugs have demonstrated that it's anything but a class effect. Specific agents have specific and unique actions.

\section{DISCLOSURES}

This article is based on a presentation funded by an educational grant from Forest Pharmaceuticals. The author discloses that he has received honoraria from Forest Pharmaceuticals for participation in this supplement. He discloses the following commercial/financial relationships through grant/research support, consultant services, speakers bureaus, and/or advisory boards: AstraZeneca, Sanofi-Aventis, King Pharmaceuticals, Novartis, and Merck.

\section{REFERENCES}

1. Chobanian AV, Bakris GL, Black HR, et al. The Seventh Report of the Joint National Committee on Prevention, Detection, Evaluation, and Treatment of High Blood Pressure: the JNC 7 report. JAMA. 2003;289:2560-72.

2. Psaty BM, Smith NL, Siscovick DS, et al. Health outcomes associated with antihypertensive therapies used as first-line agents. A systematic review and meta-analysis. JAMA. 1997;277:739-45.

3. Market Measures, The Treatment of Hypertension Study XXVI, Analytical Report 2004.

4. Hollenberg NK. The role of beta-blockers as a cornerstone of cardiovascular therapy. Am J Hypertens. 2005;18(12 pt 2):165S-168S.

5. Ubell PA, Jepson C, Asch DA. Misperceptions about beta-blockers and diuretics: a national survey of primary care physicians. J Gen Intern Med. 2003; 18:977-83.

6. Cutler JA, Neaton JD, Hulley SB, Kuller L, Paul O, Stamler J. Coronary heart disease and all-causes mortality in the Multiple Risk Factor Intervention Trial: subgroup findings and comparisons with other trials. Prev Med. 1995; 14:293-311.

7. Kostis JB, Davis BR, Cytler J, et al. Prevention of heart failure by antihypertensive drug treatment in older persons with isolated systolic hypertension. SHEP Cooperative Research Group. JAMA. 1997;278:212-16.

8. Prevention of stroke by antihypertensive drug treatment in older persons with isolated systolic hypertension. Final results of the Systolic Hypertension in the Elderly Program (SHEP). SHEP Cooperative Research Group. JAMA. 1991;265:3255-64.

9. Lewington S, Clarke R, Qizilbash N, Peto R, Collins R. Age-specific relevance of usual blood pressure to vascular mortality: a meta-analysis of individual data for one million adults in 61 prospective studies. Prospective Studies Collaboration. Lancet. 2002;360:1903-13.

10. Dalhof B, Sever PS, Poulter NR, et al. Prevention of cardiovascular events with an antihypertensive regimen of amlodipine adding perindopril as required versus atenolol adding bendroflumethiazide as required, in the Anglo-Scandinavian Cardiac Outcomes Trial-Blood Pressure Lowering Arm (ASCOT-BPLA): a multicentre randomised controlled trial. Lancet. 2005;366 (9489):895-906. 